\title{
Present and future challenge for the use of stem cell in hematopoietic transplantation and cell therapy
}

\author{
Alejandro Madrigal* \\ Department of Haematology, Royal Free and University College London, United Kingdom
}

\begin{abstract}
Hematopoietic stem cell transplantation can be a curative treatment for many diseases. Nowadays, the sources of stem cells can be several: identical sibling, cord blood, unrelated donor, and haploidentical. The HLA system displays extensive polymorphism, and the study of these antigens is now more sophisticated, making possible a more precise selection of donors. Immunotherapy has evolved, and the emerging role of different options is now a reality, including CAR-T cells. The field of immunotherapy and cellular therapy is growing fast.
\end{abstract}

Key words: Human leukocyte antigen system. Hematopoietic stem cell transplantation. Unrelated voluntary donors. Cells chimeric antigen receptor T-cells.

\section{Introduction}

Every 14 min, someone is diagnosed with blood cancer, representing 104 patients with blood cancer or related disorders every day. Hematopoietic stem cell transplantation (HSCT) can be curative treatment in a large variety of selected malignant and non-malignant diseases but relies on the availability of an appropriate donor ${ }^{1}$. Even though related donors are still the optimal source of stem cells for HSCT, $75 \%$ of the patients will not find a matching donor in their families. Hence, the initial option is to find for them an unrelated stem cell graft $^{2}$.

Over the years, Bone Marrow Donor Registries have played an important role in improving HSCT. In 1974, the world's first registry was founded by Shirley Nolan in London. Shirley's son, 3-year-old Anthony, had been diagnosed with Wiskott-Aldrich Syndrome and needed a transplant ${ }^{2}$. Following the pioneering example of the Anthony Nolan Registry, a large number of registries have now been established around the world. The growing pool of unrelated voluntary donors (VUDs), which has reached over 38 million worldwide, has contributed to the development of stem cell transplantation as a treatment method in the field of science, and over 1 million patients have been transplanted over the world to date. We have also seen an increase in the numbers of cord blood banks that have high-quality units available. The use of haploidentical donors, with new ways of conditioning and post-transplant treatment with cyclophosphamide, has helped to save the lives of many patients ${ }^{1}$.

The primary role of HLA molecules is to present peptide to $T$ cells enabling them to recognize and eliminate "foreign" particles present in an individual, as well as to prevent the recognition of "self" as foreign. The HLA system displays extensive polymorphism, most likely due to the immune system's need to keep up with and control infectious pathogens. Despite this massive diversity, we can identify donors due to the Mendelian

Date of reception: 19-10-2020

*Alejandro Madrigal

Date of acceptance: 23-10-2020

E-mail: a.madrigal@ucl.ac.uk DOI: 10.24875/RMU.M20000050 (http://creativecommons.org/licenses/by-nc-nd/4.0/)

Available online: $24-12-2020$ Medicina Universitaria. 2020;22(4):193-196 www.medicinauniversitaria.org under the CC BY-NC-ND license
} 
inheritance of HLA, the presence of well-defined haplotypes, linkage disequilibrium, and, more importantly, through the development of molecular typing techniques ${ }^{2,3}$.

Our knowledge of donor selection strategies has been enhanced considerably over the last decade by collaborative studies analyzing outcomes in very large groups of transplant patients, all with high-resolution tissue typing results. Many questions, however, remain, such as the impact of non-classical HLA loci or nonHLA genetics on clinical outcomes.

Despite data showing the need for allelic-level HLA matching, limitations in technology, capacity, and the hyper-polymorphic nature of the HLA genes have often prevented histocompatibility typing from achieving this high level of resolution.

In some difficult cases, the likelihood of identifying an unrelated donor can be estimated using various surrogate markers, such as the patient haplotype, the presence of rare alleles, or unusual HLA associations (B/C, DRB1/DQB1) and the numbers of donors identified at search ${ }^{2,3}$.

HLA matching between recipients and VUDs has remained unchanged for the past 10 years, with either an $8 / 8$ or $10 / 10$ HLA allelic match (HLA-A, -B, -C, -DRB1, and -DQB1 matched) being the preferred option $^{4}$. When analyzing the effects of matching for individual HLA mismatches on transplant outcome, it is important to consider the effect of an individual locus on an otherwise completely HLA-matched background to remove confounding effects ${ }^{2,4}$. By doing this, we found that HLA-A mismatches were tolerated with regard to all transplant outcomes analyzed, while HLA-B mismatches were associated with a highly significant survival disadvantage. HLA-C mismatches were tolerated regarding overall survival; however, they were associated with a significant increase in chronic graft versus host disease $(\mathrm{GvHD})^{4}$. The contribution of matching for HLA-DQB1 has been controversial; however, some recent studies have proved significant advantages to matching for this locus. Matching for HLA-DPB1 is particularly beneficial in reducing relapse, but due to the existence of a recombination hotspot between the HLA-DQB1 and -DPB1, allelic matching for HLA-DPB1 has been challenging ${ }^{5,6}$.

HLA typing's ideal target has always been to provide DNA typing that covered the entire gene, which is known as fully phased sequence level typing. However, for practical reasons, high-throughput methodologies have focused mainly on exons of the gene that encodes the antigen-recognition domain (ARD), with the idea that these functional regions play an important role in peptide presentation and $T$ cell recognition and, therefore, that it is critical to match them to reduce GvHD after $\mathrm{HSCT}^{5,7}$.

With the introduction of DNA typing by initially using the DNA Sanger Sequencing method, a large number of new HLA alleles have been identified that have made HLA matching between donor and patient even more difficult. In particular, because typing only for the ARD of HLA alleles cannot resolve the number of ambiguous combinations in many cases. With the introduction of new technologies that provide fully phased, high-quality DNA sequences such as NGS and third-generation sequencing (TGS), this problem seems to have been resolved. At Anthony Nolan, we were the first to introduce TGS for HLA Typing using Pacific Biosciences Single Molecule Real-Time (SMRT $\left.{ }^{\circledR}\right)$ DNA sequencing, which can sequence individual molecules of double-stranded DNA of up to 10 kilobase pairs for HLA typing. A recent study from Anthony Nolan showed, for the first time, that polymorphisms not previously detected outside the ARD regions of the HLA genes can affect the outcome of HSCT. It also illustrated that it is possible to obtain superior overall survival (OS) due to reduced non-related mortality (NRM) and GvHD risks when matching the entire genes by using full-phased high-quality DNA sequencing. The significant benefit obtained by a $12 / 12$ HLA match in survival may also reflect a higher degree of haplotype compatibility?

In conclusion, allelic/ultra-high-resolution HLA matching using full/extended gene sequencing reduces allelic ambiguity and can reveal mismatches otherwise not identified by other methods and confirmed that the selection of the ideal donor using these methods improves OS and $\mathrm{NRM}^{7}$.

\section{Immune tolerance}

One of the most important challenges in HSCT, solid organ transplantation, and autoimmune diseases has been understanding the dynamics of immune tolerance. What promotes tolerance between donor and recipient and what is the role of thymic function or the role of regulatory $T$ cells (Tregs) in promoting peripheral tolerance are still important questions that require intense investigation ${ }^{8}$.

In normal homeostasis, immune tolerance is maintained by central and peripheral mechanisms due to thymic-mediating central tolerance that leads to the removal of autoreactive T-cells and producing self-tolerant naive T-cells ${ }^{8,9}$. Tregs are a key component of 
peripheral tolerance by regulating the immune responses and limiting inflammation mediated by effector T-cells. Lack of tolerance after HSCT results in aberrant donor T-cell activity attacking the recipient, leading to significant morbidity and mortality ${ }^{9}$.

Thymic T-cell production after HSCT is essential for long-term immune reconstitution and the production of Tregs. However, thymic activity depends on the patient's age and declines gradually after the third decade of life. However, even with a highly functional thymic activity, Tregs' production can take up to 4 months, and the self-production of CD4 and CD8 cells can take a long time. How Tregs suppress or mediate the control of effector T cells to prevent or control GvHD remains unknown. My group, in the past, has developed methods to extract a large number of Tregs from umbilical cord blood with the idea to use them to treat GVHD. We have also developed a method to enumerate regulatory $T$ cells from cryopreserved umbilical cord blood samples using FOXP3 methylation-specific quantitative $\mathrm{PCR}$. We are evaluating its correlation with transplant outcome $e^{8,10}$.

\section{Immune cell therapy}

The immune cells, in particular effector T-cells and NK cell's main function, are to destroy infected cells and tumor cells. Consequently, the use of these cells for adoptive immune cell therapy has become very important in the last years. If cell therapy is considered to be the use of cells as therapeutic agents, two large areas can be differentiated, one involving regeneration, substitution, or replacement of functional cells (stem cell therapy). Moreover, the others related to the use of immune cells to exploit their specific types of responses in the effector and suppressor directions (cellular immunotherapy). In the context of allogeneic HSCT, this could lead to control of GvHD with the use of Treg cells while inducing graft versus leukemia and graft versus infection with the use of effector T-cells ${ }^{11,12}$. Another potential application, outside these areas, is regenerative medicine in the context of degenerative diseases and/or autoimmunity. Manipulation of these cells in clinical trials has shown a reduction of morbidity and mortality over other therapeutic interventions, such as surgery, chemotherapy, and radiotherapy.

Several clinical trials using effector T-cells isolated from the patient's tumor-infiltrating lymphocytes (TIL) expanded, in vitro, and reactivated with PDL-1 inhibitors' use have confirmed that these cells regain the ability to destroy the tumor cells and confirmed that these cells are capable of eliminating the tumor. However, the isolation of these specific $T$ cells to clone and expand them has proved to be highly laborious and complex ${ }^{9}$.

One of the most extraordinary advances has been using so-called "CAR-T cells" (chimeric antigen receptor T-cells). Effector T-lymphocytes normally recognize specific peptides in the context of the specific HLA molecules. However, in the case of the CART-T cells, the T-cell receptor-mediated (TCR) molecular recognition can be manipulated, using genetic engineering, to create clones of T-lymphocytes that express a transgenic chimeric receptor that recognizes unique proteins such as CD19 antigen (expressed exclusively on B-lymphocytes) $8,13,14$. Therefore, they can be used to eliminate cancer cells that regularly express this protein on their surface, such as B-line lymphoproliferative malignancies such as chronic lymphocytic leukemia (CLL). Initially, these therapies were only used against leukemias or lymphomas of the B cell line. However, at present, many clinical trials are targeting other proteins to treat not only malignant but also non-malignant conditions. Unfortunately, these therapies have several serious side effects, such as the cytokine release syndrome (CRS), that are difficult to treat and, in some cases, they can lead to high-related mortality. Despite these challenges, this is a revolutionary therapy against cancer, and it has also given impetus to the development of other immunotherapies such as the use of NK cells to produce "CAR-NK" cells using genetic engineering and similar vectors to those used in the production of CAR-T cells, with very excellent results ${ }^{8,14}$.

Finally, the use of mesenchymal cells (MSC) for cell and immunotherapy is another important line of innovative cell therapy ${ }^{9,15}$. These cells have been used extensively to treat several diseases, including autoimmune diseases such as lupus, multiple sclerosis, and cancer. The anti-inflammatory and regenerative capabilities of these cells are mediated by the secretion of cytokines and other molecules rather than through their integration or cellular replacement. There are currently over 1000 clinical studies using MSC for many different conditions and very interesting results.

In conclusion, therapeutic manipulation of the immune system may overcome morbidity and mortality caused by other therapeutic interventions such as surgery, radiotherapy, or chemotherapy. Interventions to modulate immune reactivity, such as CAR-T cells, may be valuable for the treatment of cancer, autoimmune, and chronic inflammatory disease. 


\section{Acknowledgments}

The author would like to thank the Scientific members of Anthony Nolan and Dr. Richard Duggleby for their assistance in editing the manuscript.

\section{Conflicts of interest}

The author declares no conflicts of interest.

\section{References}

1. Gratwohl A, Pasquini MC, Aljurf M, Atsuta $Y$, Baldomero $H$, Foeken $L$, et al. One million haemopoietic stem-cell transplants: a retrospective observational study. Lancet Haematol. 2015;2:91-100.

2. Madrigal JA, Scott I, Arguello R, Szydlo R, Little AM, Goldman JM. Factors influencing the outcome of bone marrow transplants using unrelated donors. Immunol Rev. 1997;157:153-66.

3. Argüello J, Little A, Pay A, Gallardo D, Genetics IR. Mutation detection and typing of polymorphic loci through double-strand conformation analysis. Nat Genet. 1998;18:192-4

4. Shaw BE, Marsh SG, Mayor NP, Russell NH, Madrigal JA. HLA-DPB1 matching status has significant implications for recipients of unrelated donor stem cell transplants. Blood. 2006;107:1220-6.

5. Shaw BE, Potter MN, Mayor NP, Pay AL, Smith C, Goldman JM, et al. The degree of matching at HLA-DPB1 predicts for acute graft-versus-host disease and disease relapse following haematopoietic stem cell transplantation. Bone Marrow Transplant. 2003;31:1001-8.

6. Petersdorf EW, Carrington M, O'huigin C, Bengtsson M, De Santis D, Dubois $V$, et al. Role of HLA-B exon 1 in graft-versus-host disease after unrelated haemopoietic cell transplantation: a retrospective cohort study. Lancet Haematol. 2020;7:50-60.

7. Mayor NP, Hayhurst JD, Turner TR, Szydlo RM, Shaw BE, Bultitude WP, et al. Recipients receiving better HLA-matched hematopoietic cell transplantation grafts, uncovered by a novel HLA typing method, have superior survival: a retrospective study. Biol Blood Marrow Transplant. 2019;25:443-5.

8. Duggleby R, Danby RD, Madrigal JA, Saudemont A. Clinical grade regulatory CD4+ T cells (Tregs): moving toward cellular-based immunomodulatory therapies. Front Immunol. 2018;9:252.

9. Saudemont A, Madrigal JA. Immunotherapy after hematopoietic stem cell transplantation using umbilical cord blood-derived products. Cancer Immunol. 2017;66:215-21.

10. Duggleby R, Tsang H, Strange K, McWhinnie A, Lamikanra A, Roberts D, et al. Enumerating regulatory $T$ cells in cryopreserved umbilical cord blood samples using FOXP3 methylation specific quantitative PCR. PLoS One. 2020;15:e0240190.

11. Duarte R, Mufti G. The effect of interleukin-7 on the alloreactivity of human $T$ cells depends on the timing of its administration in relation to the allostimulus. Bone Marrow Transplant. 2005;35:733-4.

12. Madrigal A, Rusakiewiez S, Dodi I. Immunotherapy with alloreactive T-cells? Hematol J. 2003;5:91-5.

13. De La Pena H, Madrigal JA. Cellular immunotherapy against cancer. Gac Med Oncol. 2006;5:61-4.

14. Hernández D, Madrigal A. Terapias celulares: una nueva era en la batalla contra el cáncer. Gac Med Mex. 2018;154:547-9.

15. Fernandez MN, Regidor C, Cabrera R, Garcia-Marco J, Briz M, Fores R, et al. Cord blood transplants: early recovery of neutrophils from co-transplanted sibling haploidentical progenitor cells and lack of engraftment of cultured cord. Bone Marrow Transplant. 2001;28:355-63. 